\title{
Oral Metagenomic Analysis Techniques
}

\author{
Sung-Kyun Chung ${ }^{\dagger}$ \\ Department of Dental Hygiene, Shinhan Univertsity, Uijeongbu 11644, Korea
}

\begin{abstract}
The modern era of microbial genome analysis began in earnest in the 2000s with the generalization of metagenomics and gene sequencing techniques. Studying complex microbial community such as oral cavity and colon by a pure culture is considerably ineffective in terms of cost and time. Therefore, various techniques for genomic analysis have been developed to overcome the limitation of the culture method and to explore microbial communities existing in the natural environment at the gene level. Among these, DNA fingerprinting analysis and microarray chip have been used extensively; however, the most recent method of analysis is metagenomics. The study summarily examined the overview of metagenomics analysis techniques, as well as domestic and foreign studies on disease genomics and cluster analysis related to oral metagenome. The composition of oral bacteria also varies across different individuals, and it would become possible to analyze what change occurs in the human body depending on the activity of bacteria living in the oral cavity and what causality it has with diseases. Identification, isolation, metabolism, and presence of functional genes of microorganisms are being identified for correlation analysis based on oral microbial genome sequencing. For precise diagnosis and treatment of diseases based on microbiome, greater effort is needed for finding not only the causative microorganisms, but also indicators at gene level. Up to now, oral microbial studies have mostly involved metagenomics, but if metatranscriptomic, metaproteomic, and metabolomic approaches can be taken together for assessment of microbial genes and proteins that are expressed under specific conditions, then doing so can be more helpful for gaining comprehensive understanding.
\end{abstract}

Key Words: Dental caries, Metagenomics, Microbiome, Oral

\section{Introduction}

The microbiome is defined as "all microorganisms and genes in a given environment" ${ }^{\prime 1)}$. It is possible to study the role of whole microbial communities efficiently through recent advances in next-generation sequencing (NGS).

Approximately $<1 \%$ of microorganisms are known to be culturable under general laboratory conditions ${ }^{2,3)}$. Microbes living in the human body are also unculturable, and it is estimated that approximately $20 \sim 60 \%$ of microbes cannot be cultured although it depends on the body parts ${ }^{4}$. As most microbes are not culturable (the great plate count anomaly), methods for analyzing microbial flora through culturing are no longer used ${ }^{5,6}$.

Most human microbiomes are composed of bacteria; therefore, techniques focusing on bacterial community analysis have been developed and reported in several studies. It is now possible to identify almost all species because the database for bacteria is well-established with 16S rRNA gene as the phylogenetic barcode, which also requires a taxonomy database that is accurately organized ${ }^{7}$.

The rapid development of genetic information and data analysis technologies such as NGS developed in the 2000s has led to active metagenomic studies that analyze approximately $3.9 \times 10^{13}$ symbiotic microbial communities at microbial gene level rather than through culturing.

In terms of the diagnosis and improvement of the health and treatment of disease, the human body and symbiotic microorganisms are being studied together. Research into the microbiome enables us to identify the principles of human health and disease from a new perspective and devise more fundamental and effective new treatments.

Therefore, the present study aims to investigate the analytical methods used in metagenomic studies and the 
effects that characterization of oral metagenomics could have on periodontal disease and dental caries.

\section{Materials and Methods}

This study is a review of research articles on the microbial techniques for genomic analysis and information on oral microorganisms and genomes. In stage 1 , we designed and planned research content related to the characteristics of microbial techniques for genomic analysis and information on oral microorganisms, and in stage 2 we performed a literature search. In stage 3 , based on the selected literature, we collated the research contents. In stage 4 , we objectively interpreted and derived conclusions from the analyzed data. The period for the literature search and organization of results was from 1st January to 30th April, 2019. For the literature search, we used PubMed,
KISS (Koreanstudies Information Service System), DBpia, and RISS (Research Information Sharing Service), with the search terms, 'microbiome', 'ngs', 'oral', 'metagenomic', 'subgingiva', 'caries', and 'saliva'.

\section{Results}

\section{Technology and analysis of microbiome}

\section{1) Metagenomics}

In metagenomics, DNA is extracted directly from the crude source without culturing microbes or employing a microbial isolation technique. The extracted metagenome contains a mixture of genomic DNA of all the microbes present in the sample. Its community structure is analyzed at the gene level through $\mathrm{NGS}^{8)}$. The development of metagenomics is interlocked with the commercialization

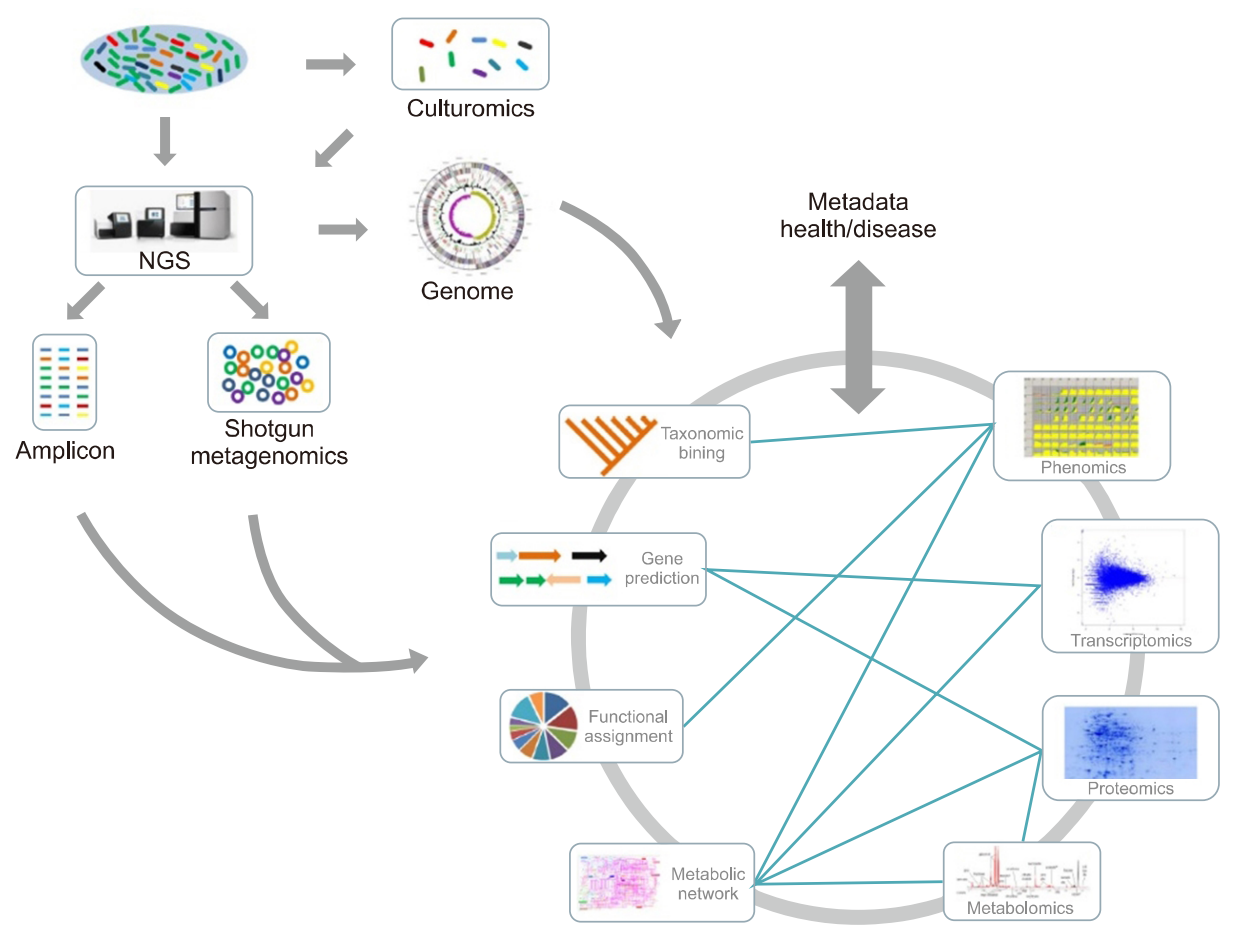

Fig. 1. The gene/genome-centric approach for the gut microbiome. Generally, 16S-rRNA based amplicon sequencing and whole shotgun sequencing are the two main metagenomic approaches for gut microbiome studies. From metagenome data, the taxonomic compositions and functional categories of the gut microbial communities, which may be associated with the health or disease state, can be inferred. Moreover, the combination of culturomics and next-generation sequencing (NGS) methods provides deeper information about the functional roles of specific gut microbial species. Other available "omics" data (transcriptomics, proteomics, metabolomics, and phenomics) provides much deeper insight into the functional role of gut microbes in human health and disease. Integrating these data with metagenomics data, especially metabolic models reconstructed from metagenomic studies, will provide a comprehensive view of metabolic interactions between microbes and host ${ }^{11}$. Adapted from the article of $\mathrm{Ji}$ and Nielsen (Front Genet 6: 219, 2015) ${ }^{12)}$ in accordance with the Creative Commons Attribution (CC BY 4.0) license. 
of NGS, which began in $2007^{9)}$. Previously, large-scale metagenomics-based studies were difficult to standardize because DNA sequencing was expensive. The purpose of metagenomics is to first determine and analyze the microbes present in the sample and second, to identify the metabolic processes and functional genes present in the sample. The shotgun (whole-genome shotgun) metagenomics approach is mainly used to view the functional gene. There is an advantage in viewing the entire metagenome, including the composition of functional genes; however, there are disadvantages too, such as the high cost of sequencing and difficulty in sequence identification ${ }^{10)}$. Conversely, to determine individual microbial species present in the diverse microbial community, an amplicon metagenomics method is used that selectively amplifies the marker gene for species identification and then analyzes the sequence of the amplification product. Thus, the effort and high-cost for analysis can be reduced greatly (Fig. 1) $)^{11,12)}$.

\section{2) Next-generation sequencing}

NGS is a DNA sequencing method that is an improvement over the existing Sanger method, and it can acquire a large number of sequences in a short time at a relatively low cost. The first NGS was achieved through Roche's 454 pyrosequencing. The method used at that time divided the entire gene into sequence fragments of $200 \mathrm{bp}$ each, which was read, and then arranged in a single line to reconstruct the whole genome code from the original 3 billion $\operatorname{codes}^{13)}$. Typical NGS technologies include Roche's 454 pyrosequencing; Illumina's sequence by synthesis; Pacific BioSciences' single molecule, real-time sequencing; and Oxford Nanopore's nanopore sequencing technologies ${ }^{14,15)}$. These technologies are far superior in throughput and cost than the existing Sanger method and are superior in read length and accuracy. NGS technologies allow the analysis of all fields of genomes across DNA, RNA, and epigenetics ${ }^{16}$. This kind of genome analysis provides a new paradigm of technology to all fields of biology and medicine by enabling the diagnosis and target treatment together (Table 1$)^{17)}$.

\section{3) 16S rRNA amplicon sequencing}

The 16S rRNA is a ribosomal RNA that constitutes a ribosome that synthesizes proteins. It has a length of 1,542 nucleotides and has a conserved region common to all species and a hypervariable region capable of classifying a specific species. The 16S rRNA gene is amplified, and the sequence is analyzed using a massive parallel method; it is a sort of an acting barcode that can identify various kinds of microbes because each microbe has a different sequence of hypervariable region. There is little mutation among the same species, but some mutations are found among different species. These differences are compared to identify the closeness between the species. If more than $97 \%$ of the 16S rRNA sequences are identical, they are the same species, if more than $94 \%$ of them are identical, they are the same genus, if more than $90 \%$ of them are identical, they are the same family, and if $85 \%$ of them are identical, they are classified as the same order ${ }^{5,18)}$.

The 16S rRNA gene is used as a marker gene to analyze the bacterial communities, and the primer used depends on the type of sequencing device. The $27 \mathrm{~F}$ and $518 \mathrm{R}$ primers are used to amplify the mutation site $\mathrm{V} 1 \sim \mathrm{V} 3$ portions when using GS Junior (Roche). The 454 technique reads a sequence backwards from the reverse primer, resulting in a sequence of $400 \sim 450 \mathrm{bp}$ in length, starting at V3 and reaching the edge of V1. In contrast, if MiSeq v3 (Illumina) is used for sequencing, 318F and 806R primers are used to amplify the $\mathrm{V} 3 \sim \mathrm{V} 4$ portions. A sequence of at least $400 \mathrm{bp}$ in length is produced by combining a pair of sequence $300 \mathrm{bp}$ in length, from both forward and reverse sides, into one through paired-end sequencing ${ }^{19)}$.

Eren et al. ${ }^{20)}$ used 16S rRNA gene sequence data from 9 areas in the oral cavity, and 493 oligomer types were identified from $\mathrm{V} 1 \sim \mathrm{V} 3$ data, and 360 oligomer types were identified from V3 $\sim$ 5 data. These types of oligomers were associated with taxonomic names at the species level by comparison with the Human Oral Microbiome Database (HOMD). They have discovered oligomer types that were sometimes differentiated by a single nucleotide that has different distributions between the oral sites and the individuals ${ }^{20)}$. 


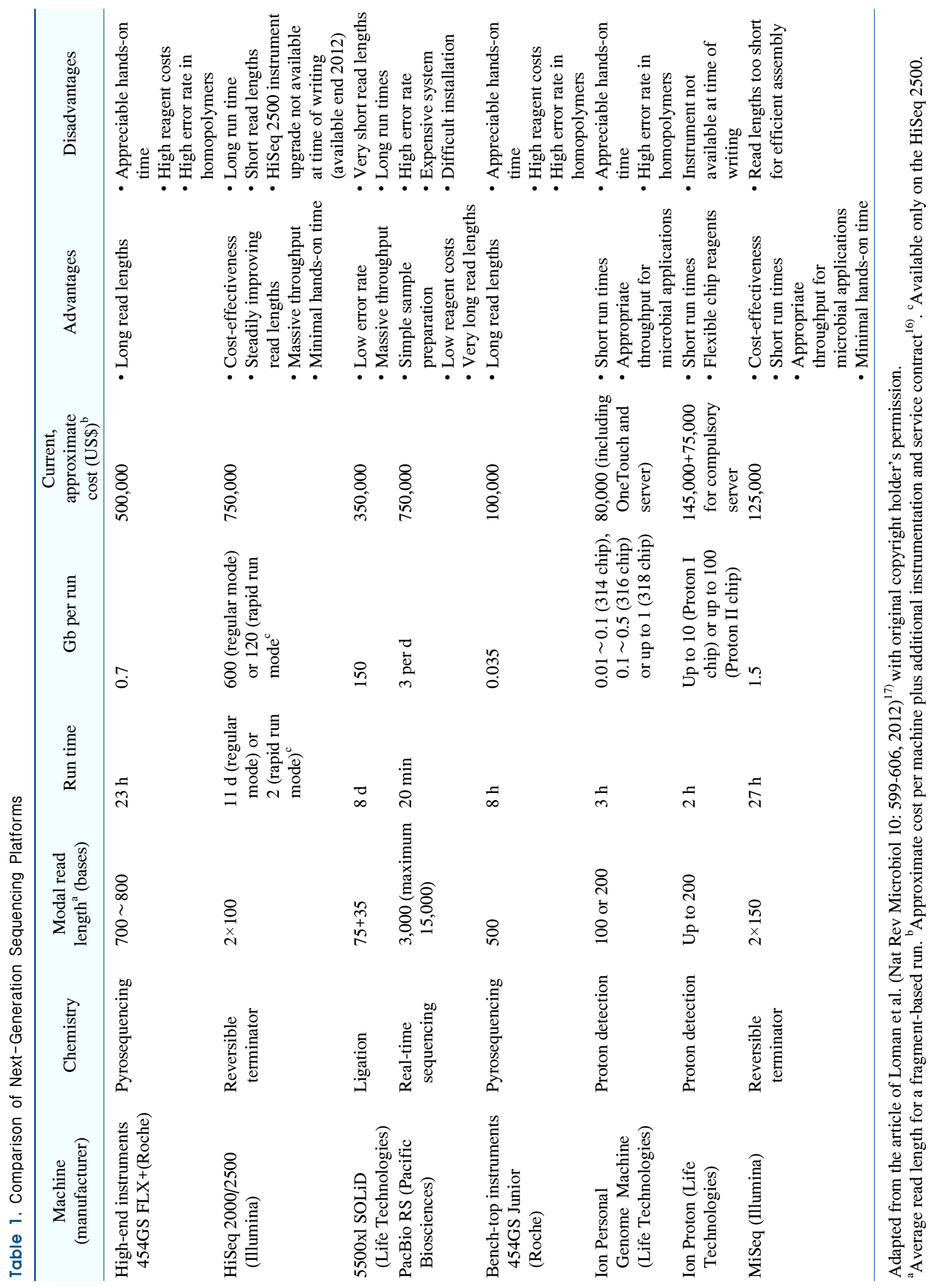




\section{4) Shotgun metagenomics sequencing}

Shotgun metagenomics sequencing is a method of analyzing functional genes in microbial communities. This is an innovative technology that mosaics the DNA extracted from the sample by a super computer after cutting it into arbitrary fragments and analyzing the sequences. The original data generated by the sequencing device contains errors that occur during the experiment and the sequencing process. The cause of errors, such as innate or inherent errors of polymerase during polymerase chain reaction (PCR), production of chimeric sequences between heterologous DNA, and homopolymer errors occurring during sequencing, are very diverse; the types of errors also depend on the experimental method or the sequencing device. These erroneous sequencing reads are filtered out, and then taxonomic assignments and diversity calculations are performed for the remaining reads. Each sequencing piece is assembled with contigs by joining the overlapped parts, and then the scaffolds are completed by listing the contigs in the order. This approach allows for the entire metagenome analysis, including the composition of complex functional genes, and has the advantage that all microbial organisms can be found at once. Currently, various bioinformatics analysis platforms have been developed, and among them, QIIME ${ }^{21)}, \mathrm{MOTHUR}^{22)}, \mathrm{RPD}^{23)}$, and PlutoF $^{24)}$ are the most frequently used programs. These platforms perform separation, identification, and diversity calculations based on code, by filtering only the sequences suitable for analysis from the original data ${ }^{25)}$.

\section{Metagenomic community analysis of oral cavity}

All techniques used for analyzing oral microbes have strengths and limitations. Based on the first selectively collected human microbial community data, the HOMD, information on microorganisms and genomes was obtained $^{26)}$. Subsequently, the $16 \mathrm{~S}$ rRNA database of the core oral microorganisms was created, which is a key phylogenetically selected core database $\mathrm{e}^{27)}$.

The HOMD includes 619 taxa in 13 phyla, as follows: Actinobacteria, Bacteroidetes, Chlamydiae, Chloroflexi, Euryarchaeota, Firmicutes, Fusobacteria, Proteobacteria, Spirochaetes, SR1, Synergistetes, Tenericutes, and TM7. Although the oral microbial phyla have been expanded to
15 , only 6 phyla account for $95 \%$ or more of the sequence, as follows: Actinobacteria, Bacteroidetes, Fusobacteria, Proteobacteria, and Spirochaetes ${ }^{28)}$.

Jorth et al. ${ }^{29)}$ confirmed the changes in the composition and gene expression of oral microbiota in healthy subjects and patients with periodontitis through expression patterns of the transcriptome. In a study conducted using samples collected from nine healthy subjects and nine patients with periodontitis, 160,000 genes were compared. The differences in the composition and gene expression of microbiota between the healthy subjects and patients with periodontitis were revealed through massively parallel RNA-sequencing, and a difference in metabolism between the two groups was observed. Conversely, the expression of genes involved in the metabolism of individual species within each microbiome was greatly diverse for each individual. In addition, disease-related microbiomes showed conservative changes in metabolism and toxic gene expression ${ }^{29)}$. Frias-Lopez and Duran-Pinedo ${ }^{30)}$ used metatranscriptome analysis for periodontal plaque in laboratory experiments and revealed that there was a rapid change in the gene expression pattern of microbiota of healthy individuals upon the addition of periodontal pathogens to the dental plaque of multi-bacterial species. Duran-Pinedo et al. ${ }^{31)}$ used subgingival microbiomes of six healthy subjects and seven patients with periodontal diseases and analyzed the in situ genome-wide transcriptome. The metabolic activities that occurred during the disease were found to be iron acquisition, lipopolysaccharide synthesis, and flagella synthesis

Wang et al. ${ }^{32)}$ described the key microbes associated with periodontitis through metagenomic sequencing. From the sequencing data of a total of 10 healthy individuals, 10 variables shared by 11 bacterial species were identified ${ }^{33)}$. Based on several literature, major genera in the mouth include: Streptococcus, Veillonella, Granulicatella, Gemella, Actinomyces, Corynebacterium, Rothia, Fusobacterium, Porphyromonas, Prevotella, Capnocytophaga, Neisseria, Haemophilus, Treponema, Lactobacterium, Eikenella, Leptotrichia, Peptostreptococcus, Staphylococcus, Eubacteria, and Propionibacterium ${ }^{34)}$.

In an integrated study conducted as part of the Human Microbiome Project (HMP) since 2012, samples were 
collected from 10 different sites in the digestive tract of more than 200 healthy individuals to compare and analyze the composition of microbiomes ${ }^{35)}$. In this study, salivary microbiota of healthy oral cavity was similar on the throat, tonsil, and the back of the tongue. The dominant phyla identified were Firmicutes, Bacteroidetes, Proteobacteria, and Fusobacteria, and they accounted for $40 \%, 25 \%$, $20 \%$, and $10 \%$, respectively. The dominant genera were found to be Streptococcus and Veillonella, and they accounted for $20 \%$ and $15 \%$ of the salivary microbiota, respectively. NGS has currently been used to examine the saliva of two adults in five data sets extracted from HMP, and more than 175 bacteria have been identified in oral saliva. Considering that the salivary microbiota is far more complex than expected, the prevalent oral microbiota of saliva is predicted to consist of approximately 900 different bacterial species ${ }^{36)}$. In another study in 2011, saliva samples from 74 children were analyzed by pyrosequencing. The occupancy rate of Firmicutes decreased as the salivary microbiota decreased from deciduous teeth to permanent teeth, but the ratio of Bacteroidetes and Proteobacteria increased as well as the total bacterial diversity $^{37)}$. Recent studies have reported that the salivary microbiota may be altered in patients with inflammatory bowel disease, emphasizing the dynamic association between microbiomes at different anatomical locations ${ }^{38)}$. In addition, it suggested that changes in local and systemic diseases lead to a structural change in the salivary microbiota $^{39)}$.

\section{Metagenomic study in the field of dental disease}

\section{1) Periodontal disease}

The virulence of the total microbiomes is increased by the core pathogen, Porphyromonas gingivalis, which interacts with secondary pathogens, like Streptococcus mitis (strain B6), and generally causes dysbiosis of the beneficial microbiota and inflammatory diseases ${ }^{40-44)}$. Every person has a group of individual-specific microorganisms that are essential for maintaining health and controlling disease. Griffen et al. ${ }^{45)}$ found differences in the microbiomes between the healthy condition and periodontitis in all phylogenetic and specific microbiomes by using $16 \mathrm{~S}$ multiple region pyrosequencing. Spirochaetes, Synergistetes, and Bacteroidetes were the dominant phyla in diseases, and a higher level of Proteobacteria appeared in healthy condition. Through this study, we were able to determine the association between $P$. gingivalis, Treponema denticola, and Tannerella forsythia and the disease. Abusleme et al. ${ }^{46)}$ performed 454 pyrosequencing using 16S rRNA gene library and confirmed that Spirochaetes, Synergistetes, Firmicutes, and Chloroflexi were higher in the microbe of periodontitis, while Actinobacteria was higher in healthy condition. Kirst et al. ${ }^{47)}$ investigated the subgingival microbiota of 25 patients with chronic periodontitis and 25 healthy subjects by using rRNA sequencing and compared them with the $\mathrm{HMP}^{48-50)}$. This study suggested that microbiota significantly changed with decreasing heterogeneity in periodontal disease. Two microbial communities were confirmed; one was Fusobacterium and Porphyromonas, which were associated with periodontitis, and the other one was Rothia and Streptococcus, which were associated with healthy condition.

Several studies have confirmed the presence of periodontal pathogens in saliva. A large-scale study in Finland used a 16S rRNA-based PCR method along with species-specific primers, where at least one of the periodontal pathogens, including T. forsythia, T. denticola, $P$. gingivalis, Campylobacter rectus, Aggregatibacter actinomycetemcomitans, and Prevotella intermedia, was found in $88 \%$ of samples in both groups of patients with periodontitis and healthy subjects ${ }^{45}$. In another study using the 16S rRNA-based PCR method, six periodontal pathogens, including Prevotella nigrescens, $A$. actinomycetemcomitans, $P$. gingivalis, $T$. forsythia, $P$. intermedia, and T. denticola, was identified in the saliva samples of 41 children with healthy oral condition. $P$. nigrescens accounted for $80 \%, T$. denticola for $32 \%, A$. actinomycetemcomitans for $24 \%$, and $P$. gingivalis for $12 \%$ of the pathogens ${ }^{51)}$. In a study in 2014 , saliva samples of a total of 586 Danish adult patients, including 139 patients with periodontitis, and 447 healthy individuals were analyzed using the human oral microbe identification microarray (HOMIM) technique, and 12 phylotypes related to periodontitis were identified in saliva ${ }^{52)}$. A recent study using $16 \mathrm{~S}$ rRNA pyrosequencing compared 
gingival plaque samples and saliva samples from 19 patients with periodontitis before and after periodontal therapy. This study showed that gingival microbiota was significantly different from salivary microbiota; the gingival microbiota significantly changed after periodontal treatment, but salivary microbiota was less affected by periodontal treatment ${ }^{53)}$.

\section{2) Dental caries}

According to the report on microbes related to caries through pyrosequencing of dental caries in samples, Streptococcus mutans was not the dominant microbe in dental caries, but a complex microbiome was involved ${ }^{54}$. This supported the previous 16S rRNA sequencing study ${ }^{55,56)}$ and the notion that dental caries were multiple bacterial diseases. In addition, through pyrosequencing, we found that oral bacteria are specific to the progression of caries $^{57)}$. Streptococcus, Granulicatella, and Actinomyces genera were significantly increased in children with severe dental caries $^{58)}$. The NGS technology, coupled with metagenomics, showed overexpression of functional genes acting as antimicrobial peptides or quorum sensing in individuals who did not experience dental caries, and thus, did not carry $S$. mutans $^{54)}$.

The analysis of the saliva of children aged $3 \sim 6$ years and samples of gingival plaque using high-throughput barcoded pyrosequencing and PCR denaturing gradient gel electrophoresis according to the presence of dental caries resulted in the identification of 156 genera belonging to 10 phyla of the saliva. In addition, salivary microbiota was reported to be different from the gingival plaque microbiota, but the genera associated with patients with dental caries have not been reported ${ }^{59)}$. Analysis of the saliva samples of 30 caries-active and 20 healthy Chinese children aged $6 \sim 8$ years by using the HOMIM technique confirmed a total of 94 taxa in 30 genera belonging to the representative 6 phyla. We were also able to confirm that 8 and 6 taxa showed a significant difference in the saliva samples from children with dental caries and healthy children, respectively ${ }^{60}$. In an integrated study using a 16S rRNA gene amplicon or whole genome-based sequencing technique to analyze saliva samples from 19 patients with dental caries and 26 healthy subjects aged $18 \sim 22$ years, salivary microbiota greatly varied in patients with dental caries compared with the healthy subjects ${ }^{61)}$. In a recent study on the expression of functional genes, functional gene microarray; HuMiChip 1.0 was used to analyze saliva samples from 10 dental caries patients and 10 healthy persons aged $18 \sim 23$ years, and we were able to confirm the association between the expression of functional gene related to disease and saliva samples from dental caries patients ${ }^{62)}$.

\section{Conclusion}

Although studies on microbial genomes have been actively conducted worldwide, the progress has been very slow and many studies still focus on how microbial imbalances lead to pathologies in human body and on their diagnosis. Recent trends in molecular biochemistry have entered the field of omics, which is a comprehensive study of all biochemical molecules that target individual genes in organisms.

As studies on large genome-wide data have currently progressed into a new phase, studies that focused on whole genome analysis, such as transcriptome sequencing and epigenome sequencing, were conducted concurrently in various large-scale groups. However, there are still limitations, such as nonrandom sampling, disease complexity, absence of large biological database, and lack of breakthrough bioinformatics analysis methods.

With the rapid development of molecular diagnostic techniques, it is important to recognize that very common oral diseases, such as periodontitis and dental caries, are multifactorial diseases and also to integrate genomics and transcriptomics to identify individuals vulnerable to diseases ${ }^{63)}$. In addition, it is necessary to focus on the functional aspect of microbiota by studying the microbiome and the complexity of the oral microbiota based on metagenomics.

\section{Notes}

\section{Conflict of interest}

No potential conflict of interest relevant to this article was reported. 


\section{Ethical approval}

It is a reviewed paper so no deliberation required.

\section{ORCID}

Sung-Kyun Chung, https://orcid.org/0000-0002-4022-1620

\section{Acknowledgements}

This work was supported by a 2017 research grant from Shinhan University, Republic of Korea.

\section{References}

1. Grice EA, Segre JA: The human microbiome: our second genome. Annu Rev Genomics Hum Genet 13: 151-170, 2012. https://doi.org/10.1146/annurev-genom-090711-163814

2. Pace NR: A molecular view of microbial diversity and the biosphere. Science 276: 734-740, 1997.

https://doi.org/10.1126/science.276.5313.734

3. Torsvik V, Goksøyr J, Daae FL: High diversity in DNA of soil bacteria. Appl Environ Microbiol 56: 782-787, 1990.

4. The NIH HMP Working Group, Jane Peterson, Susan Garges, et al.: The NIH Human Microbiome Project. Genome Res 19: 2317-2323, 2009. https://doi.org/10.1101/gr.096651.109

5. Staley JT, Konopka A: Measurement of in situ activities of nonphotosynthetic microorganisms in aquatic and terrestrial habitats. Annu Rev Microbiol 39: 321-346, 1985. https://doi.org/10.1146/annurev.mi.39.100185.001541

6. Ward DM, Weller R, Bateson MM: 16S rRNA sequences reveal numerous uncultured microorganisms in a natural community. Nature 345: 63-65, 1990. https://doi.org/10.1038/345063a0

7. Weinstock GM: Genomic approaches to studying the human microbiota. Nature 489: 250-256, 2012. https://doi.org/10.1038/nature11553

8. Handelsman J, Rondon MR, Brady SF, Clardy J, Goodman RM: Molecular biological access to the chemistry of unknown soil microbes: a new frontier for natural products. Chem Biol 5: R245-R249, 1998. https://doi.org/10.1016/S1074-5521(98)90108-9

9. Mardis ER: Next-generation DNA sequencing methods. Annu Rev Genomics Hum Genet 9: 387-402, 2008. https://doi.org/10.1146/annurev.genom.9.081307.164359

10. Kim W: Application of metagenomic techniques: understanding the unrevealed human microbiota and explaining the in clinical infectious diseases. J Bacteriol Virol 42: 263-275, 2012. https://doi.org/10.4167/jbv.2012.42.4.263

11. Woese CR, Fox GE: Phylogenetic structure of the prokaryotic domain: the primary kingdoms. Proc Natl Acad Sci U S A 74: 5088-5090, 1997. https://doi.org/10.1073/pnas.74.11.5088

12. Ji B, Nielsen J: From next-generation sequencing to systematic modeling of the gut microbiome. Front Genet 6: 219, 2015. https://doi.org/10.3389/fgene.2015.00219

13. Sogin ML, Morrison HG, Huber JA, et al.: Microbial diversity in the deep sea and the underexplored "rare biosphere". Proc Natl Acad Sci U S A 103: 12115-12120, 2006. https://doi.org/10.1073/pnas.0605127103

14. Lazarevic V, Whiteson K, Huse S, et al.: Metagenomic study of the oral microbiota by Illumina high-throughput sequencing. J Microbiol Methods 79: 266-271, 2009. https://doi.org/10.1016/j.mimet.2009.09.012

15. Gloor GB, Hummelen R, Macklaim JM, et al.: Microbiome profiling by illumina sequencing of combinatorial sequencetagged PCR products. PLoS One 5: e15406, 2010. https://doi.org/10.1371/journal.pone.0015406

16. Hummelen R, Fernandes AD, Macklaim JM, et al.: Deep sequencing of the vaginal microbiota of women with HIV. PLoS One 5: e12078, 2010. https://doi.org/10.1371/journal.pone.0012078

17. Loman NJ, Constantinidou C, Chan JZ, et al.: Highthroughput bacterial genome sequencing: an embarrassment of choice, a world of opportunity. Nat Rev Microbiol 10: 599-606, 2012. https://doi.org/10.1038/nrmicro2850

18. Janda JM, Abbott SL: $16 \mathrm{~S}$ rRNA gene sequencing for bacterial identification in the diagnostic laboratory: pluses, perils, and pitfalls. J Clin Microbiol 45: 2761-2764, 2007. https://doi.org/10.1128/JCM.01228-07

19. Klindworth A, Pruesse E, Schweer T, et al.: Evaluation of general 16S ribosomal RNA gene PCR primers for classical and next-generation sequencing-based diversity studies. Nucleic Acids Res 41: e1, 2012. https://doi.org/10.1093/nar/gks808

20. Eren AM, Borisy GG, Huse SM, Mark Welch JL: Oligotyping analysis of the human oral microbiome. Proc Natl Acad Sci U S A 111: E2875-E2884, 2014. https://doi.org/10.1073/pnas.1409644111

21. Schloss PD, Westcott SL, Ryabin T, et al.: Introducing mothur: 
open-source, platform-independent, community-supported software for describing and comparing microbial communities. Appl Environ Microbiol 75: 7537-7541, 2009. https://doi.org/10.1128/AEM.01541-09

22. Cole JR, Wang Q, Fish JA, et al.: Ribosomal database project: data and tools for high throughput rRNA analysis. Nucleic Acids Res 42: D633-D642, 2014. https://doi.org/10.1093/nar/gkt1244

23. Abarenkov K, Tedersoo L, Nilsson RH, et al.: PlutoF-a web based workbench for ecological and taxonomic research, with an online implementation for fungal ITS sequences. Evol Bioinform 6: 189-196, 2010. https://doi.org/10.4137/EBO.S6271

24. Bocci V: The neglected organ: bacterial flora has a crucial immunostimulatory role. Perspect Biol Med 35: 251-260, 1992. https://doi.org/10.1353/pbm.1992.0004

25. Hamady M, Knight R: Microbial community profiling for human microbiome projects: tools, techniques, and challenges. Genome Res 19: 1141-1152, 2009. https://doi.org/10.1101/gr.085464.108

26. Dewhirst FE, Chen T, Izard J, et al.: The human oral microbiome. J Bacteriol 192: 5002-5017, 2010. https://doi.org/10.1128/JB.00542-10

27. Griffen AL, Beall CJ, Firestone ND, et al.: CORE: a phylogenetically-curated 16S rDNA database of the core oral microbiome. PLoS One 6: e19051, 2011. https://doi.org/10.1371/journal.pone.0019051

28. Wade WG: Characterisation of the human oral microbiome. J Oral Biosci 55: 143-148, 2013. https://doi.org/10.1016/j.job.2013.06.001

29. Jorth P, Turner KH, Gumus P, Nizam N, Buduneli N, Whiteley M: Metatranscriptomics of the human oral microbiome during health and disease. MBio 5: e01012-e01014, 2014. https://doi.org/10.1128/mBio.01012-14

30. Frias-Lopez J, Duran-Pinedo A: Effect of periodontal pathogens on the metatranscriptome of a healthy multispecies biofilm model. J Bacteriol 194: 2082-2095, 2012. https://doi.org/10.1128/JB.06328-11

31. Duran-Pinedo AE, Chen T, Teles R, et al.: Community-wide transcriptome of the oral microbiome in subjects with and without periodontitis. ISME J 8: 1659-1672, 2014. https://doi.org/10.1038/ismej.2014.23

32. Wang J, Qi J, Zhao $\mathrm{H}$, et al.: Metagenomic sequencing reveals microbiota and its functional potential associated with periodontal disease. Sci Rep 3: 1843, 2013.

https://doi.org/10.1038/srep01843

33. Bik EM, Long CD, Armitage GC, et al.: Bacterial diversity in the oral cavity of 10 healthy individuals. ISME J 4: 962-974, 2010. https://doi.org/10.1038/ismej.2010.30

34. Zarco MF, Vess TJ, Ginsburg GS: The oral microbiome in health and disease and the potential impact on personalized dental medicine. Oral Dis 18: 109-120, 2012. https://doi.org/10.1111/j.1601-0825.2011.01851.x

35. Segata N, Haake SK, Mannon P, et al.: Composition of the adult digestive tract bacterial microbiome based on seven mouth surfaces, tonsils, throat and stool samples. Genome Biol 13: R42, 2012. https://doi.org/10.1186/gb-2012-13-6-r42

36. Hasan NA, Young BA, Minard-Smith AT, et al.: Microbial community profiling of human saliva using shotgun metagenomic sequencing. PLoS One 9: e97699, 2014. https://doi.org/10.1371/journal.pone.0097699

37. Crielaard W, Zaura E, Schuller AA, Huse SM, Montijn RC, Keijser BJ: Exploring the oral microbiota of children at various developmental stages of their dentition in the relation to their oral health. BMC Med Genomics 4: 22, 2011. https://doi.org/10.1186/1755-8794-4-22

38. Said HS, Suda W, Nakagome S, et al.: Dysbiosis of salivary microbiota in inflammatory bowel disease and its association with oral immunological biomarkers. DNA Res 21: 15-25, 2014. https://doi.org/10.1093/dnares/dst037

39. Ding T, Schloss PD: Dynamics and associations of microbial community types across the human body. Nature 509: 357-360, 2014. https://doi.org/10.1038/nature13178

40. Kim AR, Jeong MJ, Ahn YS, Kim MN, Kim SI, Lim DS: The interactive effect of these bacterial substrates on the growth of Streptococcus gordonii, Fusobacterium nucleatum and Porphyromonas gingivalis. J Dent Hyg Sci 15: 209-219, 2015. https://doi.org/10.17135/jdhs.2015.15.2.209

41. Hwang SJ: Influence of smoking cessation on periodontal biomarkers in gingival crevicular fluid for 1 year: a case study. J Dent Hyg Sci 14: 525-536, 2014.

https://doi.org/10.17135/jdhs.2014.14.4.525

42. Choi ES, Cho HA: Association between oral health status and rheumatoid arthritis. J Dent Hyg Sci 15: 612-619, 2015. https://doi.org/10.17135/jdhs.2015.15.5.612

43. Hajishengallis G, Lamont RJ: Beyond the red complex and 
into more complexity: the polymicrobial synergy and dysbiosis (PSD) model of periodontal disease etiology. Mol Oral Microbiol 27: 409-419, 2012. https://doi.org/10.1111/j.2041-1014.2012.00663.x

44. Hajishengallis G, Darveau RP, Curtis MA: The keystonepathogen hypothesis. Nat Rev Microbiol 10: 717-725, 2012. https://doi.org/10.1038/nrmicro2873

45. Griffen AL, Beall CJ, Campbell JH, et al.: Distinct and complex bacterial profiles in human periodontitis and health revealed by $16 \mathrm{~S}$ pyrosequencing. ISME J 6: 1176-1185, 2012. https://doi.org/10.1038/ismej.2011.191

46. Abusleme L, Hong BY, Dupuy AK, Strausbaugh LD, Diaz PI: Influence of DNA extraction on oral microbial profiles obtained via 16S rRNA gene sequencing. J Oral Microbiol 6: e1-e7, 2014. https://doi.org/10.3402/jom.v6.23990

47. Kirst ME, Li EC, Alfant B, et al.: Dysbiosis and alterations in predicted functions of the subgingival microbiome in chronic periodontitis. Appl Environ Microbiol 81: 783-793, 2015. https://doi.org/10.1128/AEM.02712-14

48. Turnbaugh PJ, Ley RE, Hamady M, Fraser-Liggett CM, Knight R, Gordon JI: The human microbiome project: exploring the microbial part of ourselves in a changing world. Nature 18: 449: 804-810, 2007. https://doi.org/10.1038/nature06244

49. Abusleme L, Dupuy AK, Dutzan N, et al.: The subgingival microbiome in health and periodontitis and its relationship with community biomass and inflammation. ISME $\mathrm{J} 7$ : 1016-1025, 2013. https://doi.org/10.1038/ismej.2012.174

50. Könönen E, Paju S, Pussinen PJ, et al.: Population-based study of salivary carriage of periodontal pathogens in adults. $\mathrm{J}$ Clin Microbiol 45: 2446-2451, 2007. https://doi.org/10.1128/JCM.02560-06

51. Kulekci G, Leblebicioglu B, Keskin F, Ciftci S, Badur S: Salivary detection of periodontopathic bacteria in periodontally healthy children. Anaerobe 14: 49-54, 2008. https://doi.org/10.1016/j.anaerobe.2007.08.001

52. Belstrøm D, Fiehn NE, Nielsen $\mathrm{CH}$, et al.: Differences in bacterial saliva profile between periodontitis patients and a control cohort. J Clin Periodontol 41: 104-112, 2014. https://doi.org/10.1111/jcpe.12190
53. Yamanaka W, Takeshita T, Shibata Y, et al.: Compositional stability of a salivary bacterial population against supragingival microbiota shift following periodontal therapy. PLoS One 7: e42806, 2012. https://doi.org/10.1371/journal.pone.0042806

54. Belda-Ferre $\mathrm{P}$, Alcaraz LD, Cabrera-Rubio R, et al.: The oral metagenome in health and disease. ISME J 6: 46-56, 2012. https://doi.org/10.1038/ismej.2011.85

55. Corby PM, Lyons-Weiler J, Bretz WA, et al.: Microbial risk indicators of early childhood caries. J Clin Microbiol 43: 5753-5759, 2005.

https://doi.org/10.1128/JCM.43.11.5753-5759.2005

56. Aas JA, Griffen AL, Dardis SR, et al.: Bacteria of dental caries in primary and permanent teeth in children and young adults. J Clin Microbiol 46: 1407-1417, 2008. https://doi.org/10.1128/JCM.01410-07

57. Jiang $\mathrm{W}$, Ling $\mathrm{Z}$, Lin $\mathrm{X}$, et al.: Pyrosequencing analysis of oral microbiota shifting in various caries states in childhood. Microb Ecol 67: 962-969, 2014. https://doi.org/10.1007/s00248-014-0372-y

58. Jiang W, Zhang J, Chen H: Pyrosequencing analysis of oral microbiota in children with severe early childhood dental caries. Curr Microbiol 67: 537-542, 2013. https://doi.org/10.1007/s00284-013-0393-7

59. Ling Z, Kong J, Jia P, et al.: Analysis of oral microbiota in children with dental caries by PCR-DGGE and barcoded pyrosequencing. Microb Ecol 60: 677-690, 2010. https://doi.org/10.1007/s00248-010-9712-8

60. Luo AH, Yang DQ, Xin BC, Paster BJ, Qin J: Microbial profiles in saliva from children with and without caries in mixed dentition. Oral Dis 18: 595-601, 2012. https://doi.org/10.1111/j.1601-0825.2012.01915.x

61. Yang $F$, Zeng $X$, Ning $K$, et al.: Saliva microbiomes distinguish caries-active from healthy human populations. ISME J 6: 1-10, 2012. https://doi.org/10.1038/ismej.2011.71

62. Yang F, Ning K, Chang X, et al.: Saliva microbiota carry caries-specific functional gene signatures. PLoS One 9: e76458, 2014. https://doi.org/10.1371/journal.pone.0076458

63. Ai JY, Smith B, Wong DT: Bioinformatics advances in saliva diagnostics. Int J Oral Sci 4: 85-87, 2012. https://doi.org/10.1038/ijos.2012.26 